\title{
Analysis and Design of Double-mitered Right-angle Bend based on Planar Circuit Model and Rigorous Equivalent Network
}

\author{
Yasuhiro AKIMOTO Takaharu HIRAOKA Jui-Pang HSU \\ Faculty of Engineering, Kanagawa University, Yokohama Japan
}

Abstract-Strip-line right-angle bend is a key component for MIC. It has been demonstrated that right-angle bend with proper cut can improve the frequency characteristics of no-cut right-angle bend. However their characteristics are deteriorated with frequency, which can be improved again by double-mitered structure. In this paper how to analyze the double-mitered right-angle bend is explained based on 2-D planar circuit model and rigorous equivalent network. The analysis demonstrates the mechanism of no reflection and guideline for design. Also effective equivalent network of 45 degree planar junction for dominant mode will work well for analysis and design of the double-mitered structure. Finally dynamic behavior of 2-D field distribution is calculated and shown, which will help to understand the operation of double-mitered bend.

Keywords- Stripline double-mitered right-angle bend, 45 degree planar junction, eigenvalue, Foster-type equivalent network, equivalent network for dominant mode, phase characteristic

\section{INTRODUCTION}

Stripline right-angle bend is one of the key components for MIC. Analysis by 2-D planar circuit model ${ }^{[1]}$ demonstrates that frequency characteristics of right-angle bend with no cut shown in Fig.1( $a$ ) can be improved to realize low reflection and wider band-width by square-shaped cut shown in Fig.1(b $)^{[2]}$ and improved further by slantwise cut as shown in Fig.1(c) ${ }^{[3],[4]}$. However, their frequency characteristics are deteriorated with frequency and these structure can not realize low reflection at higher frequency. Double-mitered bend as shown in Fig.1 $(d)$ can solve this problem; reflection by first 45 degree planar junction can be cancelled by the reflection caused by second junction, resulting in no reflection at all. So far how to design the structure (determination of the length of middle strip-line connecting two mitered bends) is not clear. The reason is that performance of single mitered bend (45 degree) is roughly known ${ }^{[5],[6]}$, but effect of coupling between two discontinuities is not known. Hence in this paper rigorous equivalent network is introduced based on planar circuit model, and is applied to the calculation of the frequency characteristics and analysis of operation, through which effect of two discontinuities and mechanism of no-reflection at input port are investigated. The results of these analysis will give how to determine the length of strip-line for realizing no reflection at the desired frequency. Also equivalent network for single mitered bend (45 degree) is introduced and shown to be useful for not short strip-line case where higher mode coupling can be neglected. Finally, dynamic 2-D field distribution is calculated and demonstrated. Through the analysis $W=W_{0}$ in Fig. $2(a)$ is assumed.

\section{Equivalent Network By Mode THEORY}

Stripline double-mitered right-angle bend shown in Fig.2(a) can be modelled to planar circuit with magnetic side wall. Then, the structure is divided into three planar waveguides and two 45 degree planar junctions. Equivalent network for planar waveguide is given by multi-transmission line as in Fig.2(b) whose network parameters (mode propagation constant and mode characteristic

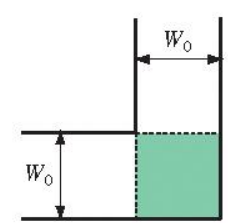

(a) Not cut
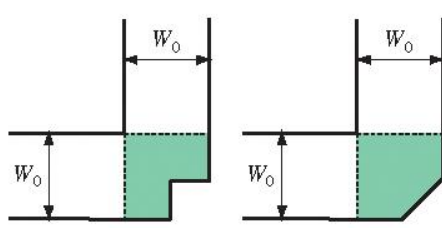

(b) Square-shaped cut (c) Slantwise cut (d) Double-mitered Fig. 1 Various stripline right-angle bend

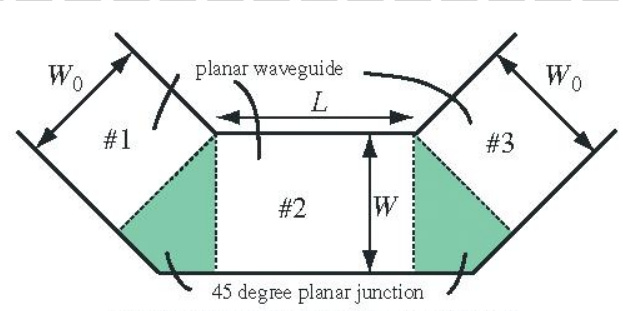

(a) Double-mitered right-angle bend

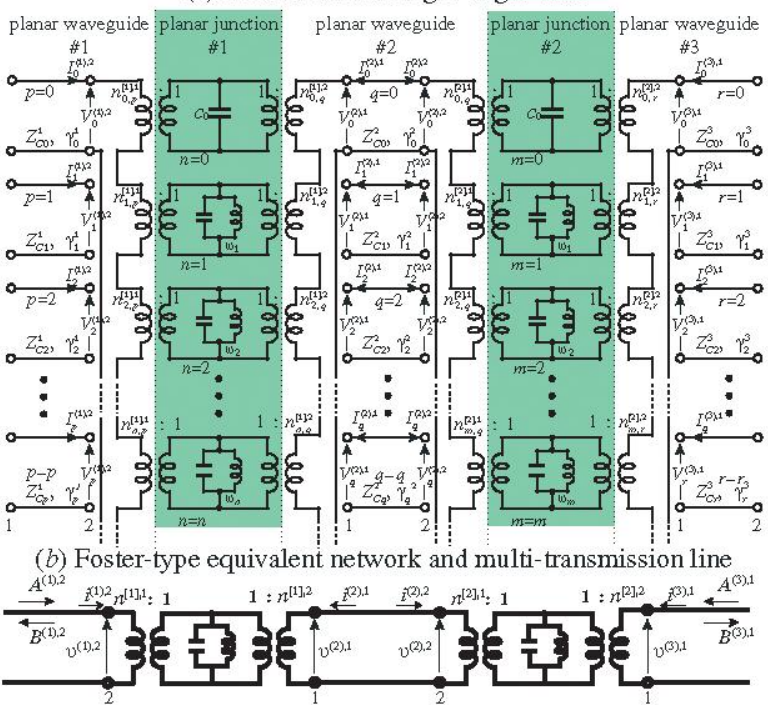

(c) Vector notation

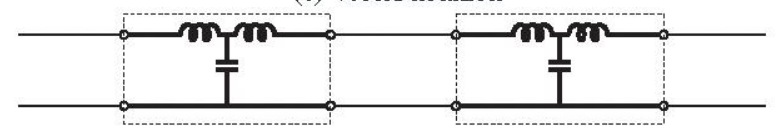

(d) Equivalent network for dominant mode

Fig. 2 Right-angle bend and its equivalent network

Table 1 Network parameters for equivalent network

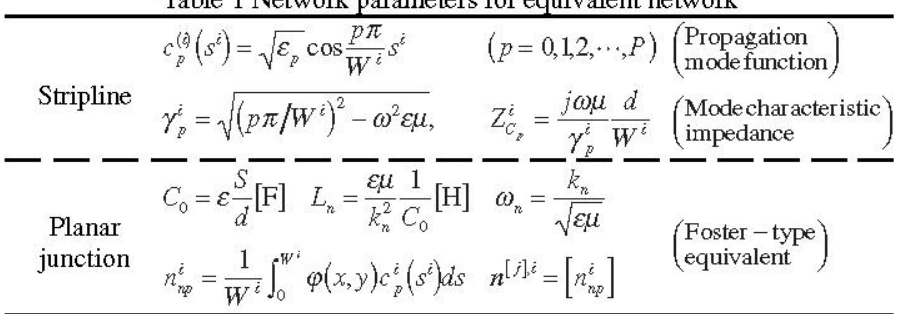


impedance) are given in Table 1. Foster-type equivalent network for planar junctions is also shown in Fig.2(b), whose equivalent network parameters are derived by solving eigenmode of the corresponding 45 degree planar junction and given by Table 2 . Therefore whole equivalent network for stripline double-mitered rightangle bend is given by Fig.2(b) or Fig.2(c) in vector notation. By the way eigenmode of the 45 degree planar junction has been solved by 3 -stage configuration and double symmetry with magnetic wall ${ }^{[4]}$. Eigenmodes up to 60 th are calculated, whose eigenvalues are in Table 2 and field distribution $\varphi_{n}(x, y)$ of lower eigenmodes up to 10th are shown in Fig.3. Also whole equivalent network for dominant mode approximation is shown in Fig.2(d), where equivalent network of single 45 degree planar junction given by T-type networks ${ }^{[6]}$ is used.

\section{Circuit ANALysis For Right-ANGLE BEND}

Double-mitered right-angle bend is analyzed by equivalent network given in Fig.2(c) and circuit theory, where mode voltage/ current column vector of $j$-th port in $i$-th planar waveguide are defined as $\boldsymbol{v}^{(i), j}, \boldsymbol{i}^{(i), j}$ in order to consider higher mode of planar waveguide. Then, following matrix relation (1) is given among mode voltage/current column vector of two planar junctions based on mode impedance matrix of 45 degree planar junction, whose element is given by eq.(2).

$$
\begin{aligned}
& {\left[\begin{array}{l}
\boldsymbol{v}^{(1), 2} \\
\boldsymbol{v}^{(3), 1} \\
\boldsymbol{v}^{(2), 1} \\
\boldsymbol{v}^{(2), 2}
\end{array}\right]=\left[\begin{array}{cccc|c}
Z^{1,1} & 0 & Z^{1,2} & 0 & \vec{i}^{(1), 2} \\
0 & Z^{2,2} & 0 & Z^{2,1} & \bar{i}^{(3), 1} \\
Z^{2,1} & 0 & Z^{2,2} & 0 & \breve{i}^{(2), 1} \\
0 & Z^{1,2} & 0 & Z^{1,1} & \vec{i}^{(2), 2}
\end{array}\right]} \\
& Z^{i, j}=\left(Z_{p, q}^{i, j}\right) \quad Z_{p, q}^{i, j}=j \frac{1}{C_{0}} \sum_{n=0}^{N} \frac{\omega}{\omega^{2}-\omega_{n}^{2}} \cdot n_{n p}^{i} \cdot n_{n q}^{j}
\end{aligned}
$$

where $Z^{1,1}=Z^{2,2}, Z^{1,2}=\left(Z^{2,1}\right)^{t}$

When external mode voltage/current matrix $\boldsymbol{v}^{e}, \boldsymbol{i}^{e}$ and internal mode voltage/current matrix $\boldsymbol{v}^{i}, \boldsymbol{i}^{i}$ are introduced by eq.(3), eq.(1) is transformed to eq.(4).

$$
\begin{aligned}
& \boldsymbol{v}^{e}=\left[\begin{array}{l}
\boldsymbol{v}^{(1), 2} \\
\boldsymbol{v}^{(3), 1}
\end{array}\right] \quad \boldsymbol{i}^{e}=\left[\begin{array}{l}
\vec{i}^{(1), 2} \\
\tilde{i}^{(3), 1}
\end{array}\right] \quad \mathbf{v}^{i}=\left[\begin{array}{l}
\mathbf{v}^{(1), 2} \\
\mathbf{v}^{(3), 1}
\end{array}\right] \quad i^{i}=\left[\begin{array}{l}
\tilde{i}^{(2), 1} \\
\vec{i}^{(2), 2}
\end{array}\right] \\
& {\left[\begin{array}{c}
\mathbf{v}^{e} \\
\boldsymbol{v}^{i}
\end{array}\right]=\left[\begin{array}{ll}
Z^{e e} & Z^{e i} \\
Z^{i e} & Z^{i i}
\end{array}\right]\left[\begin{array}{l}
i^{e} \\
i^{i}
\end{array}\right]}
\end{aligned}
$$

Moreover, the internal mode voltage and current is related by \#2 planar waveguide which gives following relation through transmission line theory.

$$
\begin{aligned}
& v^{i}=-Z^{i} i^{i} \\
& Z^{i}=\left[\begin{array}{ll}
Z_{C}^{(2)} \operatorname{coth} \gamma^{(2)} L & Z_{C}^{(2)} \operatorname{csch} \gamma^{(2)} L \\
Z_{C}^{(2)} \operatorname{csch} \gamma^{(2)} L & Z_{C}^{(2)} \operatorname{coth} \gamma^{(2)} L
\end{array}\right]
\end{aligned}
$$

Therefore, the effective external impedance matrix $Z_{e f f}^{e}$ is given by eq.(6).

$$
\boldsymbol{v}^{e}=\left[Z^{e e}-Z^{e i}\left(Z^{i i}+Z^{i}\right)^{-1} Z^{i e}\right] i^{e}=Z_{e f f}^{e} \dot{i}^{e}
$$

When incident and reflected mode voltage matrix at external $\mathrm{I} / \mathrm{O}$ ports in Fig.2(c) $\boldsymbol{A}^{e}, \boldsymbol{B}^{e}$ are defined by eq.(7), then external mode voltage/current matrix are given by eq.(8).

$$
\begin{aligned}
& \boldsymbol{A}^{e}=\left[\begin{array}{l}
\boldsymbol{A}^{(1), 2} \\
\boldsymbol{A}^{(3), 1}
\end{array}\right] \quad \boldsymbol{B}^{e}=\left[\begin{array}{l}
\boldsymbol{B}^{(1), 2} \\
\boldsymbol{B}^{(3), 1}
\end{array}\right] Y_{C}^{e}=\operatorname{diag}\left(Y_{C}^{(1)}, \boldsymbol{Y}_{C}^{(3)}\right) \\
& \boldsymbol{v}^{e}=\boldsymbol{A}^{e}+\boldsymbol{B}^{e} \quad i^{e}=Y_{C}^{e}\left(\boldsymbol{A}^{e}-\boldsymbol{B}^{e}\right)
\end{aligned}
$$

Substituting eq.(8) into eq.(9) and reorganizing them, voltage scat-
Table 2 Eigenvalue of planar junction $\left(W=W_{0}\right) \quad k_{n} W_{0}=\omega_{n} \sqrt{\varepsilon \mu} W_{0}$

\begin{tabular}{|c|c|c|c|c|c|c|c|}
\hline 1 & 3.726576563 & 16 & 19.09301094 & 31 & 27.29351991 & 46 & 34.30639833 \\
\hline 2 & 5.446114404 & 17 & 19.0967201 & 32 & 28.38061073 & 47 & 34.70798204 \\
\hline 3 & 6.789571595 & 18 & 20.5010881 & 33 & 28.41707264 & 48 & 34.7843416 \\
\hline 4 & 9.027583132 & 19 & 21.40425922 & 34 & 29.12963596 & 49 & 35.49455031 \\
\hline 5 & 9.250266053 & 20 & 22.07462668 & 35 & 29.32690883 & 50 & 35.65154293 \\
\hline 6 & 10.0708373 & 21 & 22.10930898 & 36 & 29.6049937 & 51 & 35.9743169 \\
\hline 7 & 12.56658407 & 22 & 22.50031776 & 37 & 30.61250935 & 52 & 36.09227564 \\
\hline 8 & 12.81142971 & 23 & 22.52957383 & 38 & 31.19492642 & 53 & 37.45090533 \\
\hline 9 & 13.64716352 & 24 & 23.84474336 & 39 & 31.22687865 & 54 & 37.50927488 \\
\hline 10 & 13.74382837 & 25 & 25.05573413 & 40 & 31.61357747 & 55 & 37.78382989 \\
\hline 11 & 15.84601346 & 26 & 25.1509394 & 41 & 31.6145695 & 56 & 37.81060305 \\
\hline 12 & 15.89023485 & 27 & 25.45142901 & 42 & 32.54473153 & 57 & 38.28903558 \\
\hline 13 & 16.8798096 & 28 & 25.75381158 & 43 & 32.6049875 & 58 & 38.28924044 \\
\hline 14 & 17.82271497 & 29 & 26.59629803 & 44 & 33.5000477 & 59 & 39.26135858 \\
\hline 15 & 18.24767022 & 30 & 26.99355941 & 45 & 34.15949315 & 60 & 39.29778443 \\
\hline
\end{tabular}

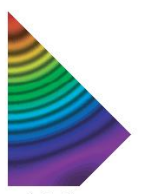

(a) 1 st

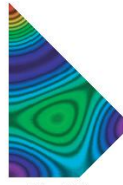

(f) 6 th

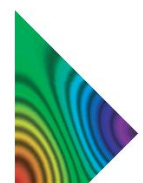

(b) 2nd

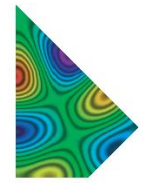

(g) 7 th
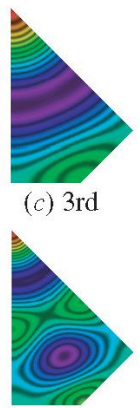

(h) 8 th

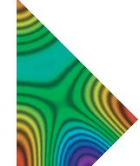

(d) 4 th

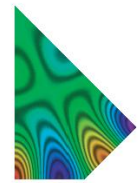

(i) 9 th

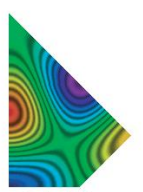

(e) 5 th

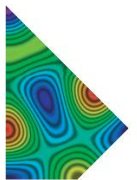

(j) 10 th
Fig. 3 Field distribution of eigenmode for 45 degree bend

tering matrix which is defined as $\boldsymbol{B}^{e}=\boldsymbol{S}_{V} \boldsymbol{A}^{e}$ is given by eq.(9).

$$
S_{V}=\left(Z_{e f f}^{e} Y_{C}^{e}+I\right)^{-1}\left(Z_{e f f}^{e} Y_{C}^{e}-I\right)
$$

From this equation mode voltage scattering matrix for dominant mode $S_{v 1,1}$ is derived, which leads to give mode voltage scattering matrix for dominant mode as $\boldsymbol{S}_{P 1,1}=\boldsymbol{S}_{V 1,1}$.

\section{Frequency Characteristics And Field Distribution}

Frequency characteristics of the double-mitered right-angle bend are calculated based on rigorous equivalent network of Fig.2(c) and eqs.(6) and (9). Width of the three planar waveguides is $W_{0}=3.37[\mathrm{~mm}]$ and relative dielectric constant is $\varepsilon_{r}=2.62$. Calculated frequency characteristics of the power transmission coefficient $S_{P 1,1}^{2,1}$ in $[\mathrm{dB}]$ up to $25[\mathrm{GHz}]$ are shown in Figs.4 and $6(\alpha)$ with connecting stripline length as a parameter. Through this calculation up to 60 th eigenmode of 45 degree planar junction and up to 20th propagation mode of the planar waveguide is taken into consideration. It turns out that double-mitered bend realizes complete transmission at certain discrete frequencies. Also frequency characteristics are calculated based on equivalent network for dominant mode given in Fig.2(d) and shown by dotted line in the same figures for comparison. It turns out that calculated results by dominant mode approximation agree well with exact results for longer stripline but not for $1.0[\mathrm{~mm}]$ length. The reason is dominant mode approximation neglects the higher mode coupling and its effect becomes large with shortness of the stripline. 2-D field distribution at operation of no reflection (A) with time phase is calculated and shown in Fig.5. 


\section{ANALYSIS OF OPERATION}

From the calculated results of Fig.4 or Fig.6( $a)$ double-mitered right-angle bend realizes complete transmission at certain discrete frequencies and these discrete frequencies become lower with length of the connecting stripline. This result can be explained by interference cancellation between the reflection of first 45 degree planar junction and that of the second planar junction in double-mitered bend. In order to investigate operating mechanism in detail, practical situation at operation for middle stripline length of $L=5.0[\mathrm{~mm}]$ is analyzed in the following.

5-1 The exact frequency characteristics of reflection and transmission for $L=5.0[\mathrm{~mm}]$ case shown in Fig.6 $(a)$ has two complete transmission at frequency (A) $7.53[\mathrm{GHz}]$ and (C) $21.72[\mathrm{GHz}]$, and relatively low transmission at frequency (B) $16.55[\mathrm{GHz}]$ whose transmission and reflection coefficient are in Table 3.

5-2 Incident and reflected mode amplitude including higher mode at each port (Fig.6(b)) for unit amplitude incidence of dominant mode at \#1 waveguide are calculated and shown in Fig.6(c) with mode order. TEM mode order is " 0 ". It turns out that incident amplitude of higher modes at each port of waveguide \#2 is very small because of evanescent attenuation, but reflected amplitude of higher mode is not small because of discontinuity excitation.

5-3 No reflection and complete transmission due to interference cancellation will be proved by practical analysis at (A) and (C) shown in Fig. 6(a). Total reflected amplitude at port 2 of \#1 waveguide consists of reflection $\boldsymbol{R}_{1}$ by first junction and reflection $\boldsymbol{R}_{2}$ by second junction as shown in Fig.7 $(a) . \boldsymbol{R}_{1}$ is estimated by frequency characteristics of single 45 degree planar junction shown in Fig. $7(b)^{[6]}$. $\boldsymbol{R}_{2}$ (a) is estimated based on Fig.6(b) where multimode coupling is taken into consideration. Estimated results of $\boldsymbol{R}_{1}$ and $\boldsymbol{R}_{2}$ are plotted in Fig.7 $(c)$ for three frequencies (A,B,C), whose numerical value are in Table 4 including sum of $\boldsymbol{R}_{1}$ and $\boldsymbol{R}_{2}$. From these results we can see that first reflection is cancelled out by second reflection for case (A), (C) to realize no reflection because their amplitude is the same but their phase 180 degrees difference. Thus complete transmission is proved to be due to the interference cancellation. Also $\boldsymbol{R}_{2}$ is estimated by equivalent network for dominant mode approximation given by Fig2 $(d)$, whose result is given by eq.(10)

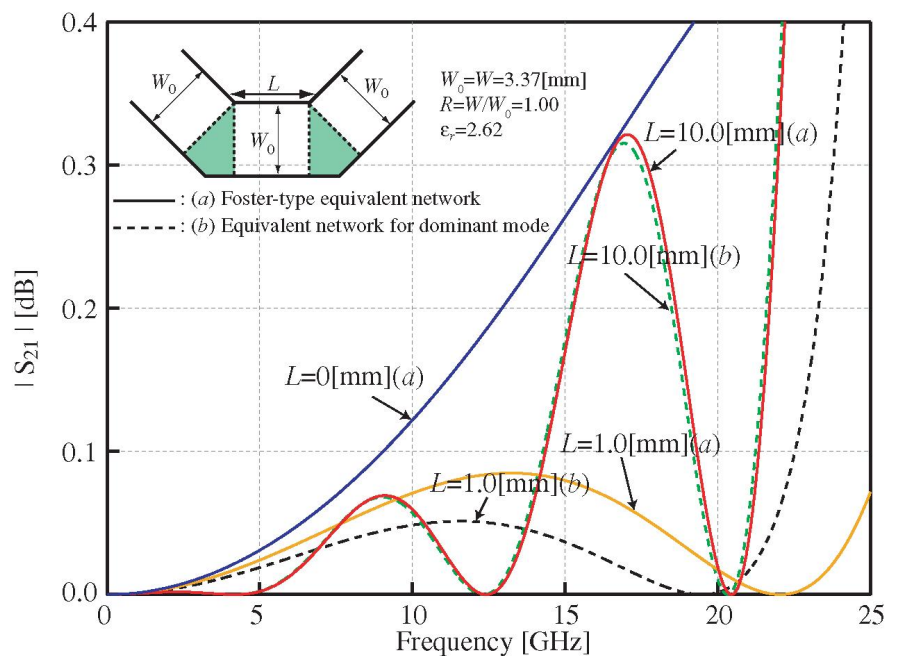

Fig.4 Frequency characteristics for right-angle bend for $L=0, L=1.0, L=10.0[\mathrm{~mm}]$

$$
\boldsymbol{R}_{2}=\frac{1-\left(R^{2} \exp (-j 2 \beta L)\right)^{N}}{1-R^{2} \exp (-j 2 \beta L)} T^{2} R \exp (-j 2 \beta L)
$$

where $N$ is number of path through planar waveguide. $\boldsymbol{R}_{2}$ of stationary case ( $N=$ infinity) for case $(\mathrm{A})$ is calculated and shown in Fig.7(c) by $\boldsymbol{R}_{2}$ (b). It is interesting that $\boldsymbol{R}_{2}$ (a) of multimode analysis and $\boldsymbol{R}_{2}$ (b) of dominant mode analysis is almost the same, which is used the bend design.

5-4 Guideline for design can be given by the previous analysis, which demonstrates that equivalent network for dominant mode is useful. By the way $\boldsymbol{R}_{2}$ is again estimated by single path $(N=1)$ and infinite path ( $N=$ infinity) for case (A) and plotted Fig. $7(c)$, which shows they are almost equal. Hence $\boldsymbol{R}_{2}$ is roughly estimated by single path $(N=1)$. Then the phase difference of $\boldsymbol{R}_{1}$ and $\boldsymbol{R}_{2}$ is estimated by the following equation, which can determine the stripline length for the desired center frequency.

$$
\text { Phase difference }=2(\angle T+\beta L)
$$

\section{CONCLUSION}

It is well-known that double-mitered right-angle bend can realize better transmission compared with other right-angle bend structure. However how to design it is not clear because of two dimensional field problem. Rigorous equivalent network for the double-mitered right-angle bend is formulated by planar circuit model, and applied to the calculation of practical structure and analysis of operation. The analysis demonstrates that the cancellation of reflection by two discontinuities works properly. This analysis show that how to design the double-mitered right-angle bend at the desired center frequency.

\section{Reference}

[1]A.A.Oliner "Equuivalent circuits for discontinuities in balanced strip transmission line" pp1134-143 IEEE MTT vol (1954)

[2]T.Hiraoka, Y. Tabei, K.Kojima, J.P.Hsu "Analysis of stripline right-angle bend with square-shaped corner cut based on eigenmode expansion method and Foster-type equivalent network" APMC'98 WE1B-3

[3]Hsu,J.P.,T.Hiraoka,Y. Tabei " Analysis of stripline right-angle bend with slant-wise corner cut based on eigenmode expansion method and Foster-type equivalent network" IEEE IMS1999 WEF2-2

[4]T.Hiraoka, Hsu,J.P. "Analysis of Stripline right-angle bend with slantwise corner cut based on Foster-type equivalent network and exact calulation of eigenmode" APMC2001 WE1C-5 pp449-452

[5]T.Hiraoka,K.Hamatani, J.P.Hsu "Foster-type equivalent network for strip line 45 degrees bend based on calculation of eigenmode and its application" CJMW2004 A-1-10 pp33-36

[6]T.Hiraoka,K.Hamatani, J.P.Hsu "Calculation of Foster-type equivalent network for stripline 45 degrees bend based on novel calculation method of the eigenmode" EuMC2005
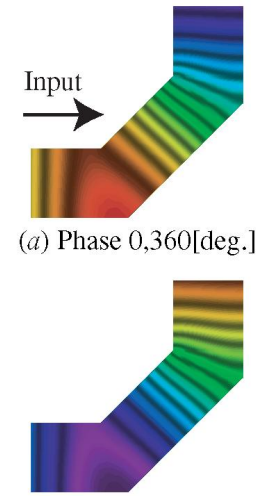

(d) Phase 180 [deg.]

Fig.5 Field distributi (a) Phase 0,360[deg.]

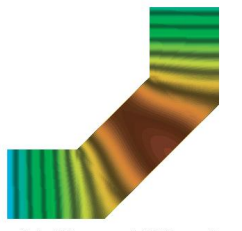

(b) Phase 60[deg.]

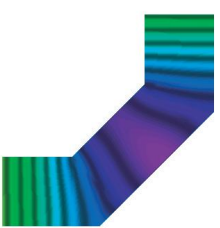

(e) Phase 240[deg.]

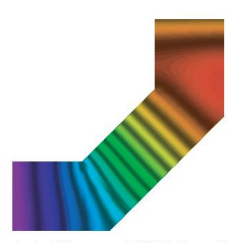

(c) Phase 120 [deg.]

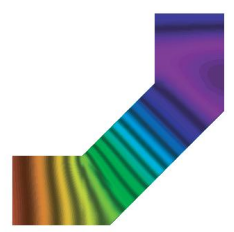

(f) Phase 300 [deg.]
on with time phase
$(L=5.0[\mathrm{~mm}], \mathrm{F}=7.53[\mathrm{GHz}])$ 


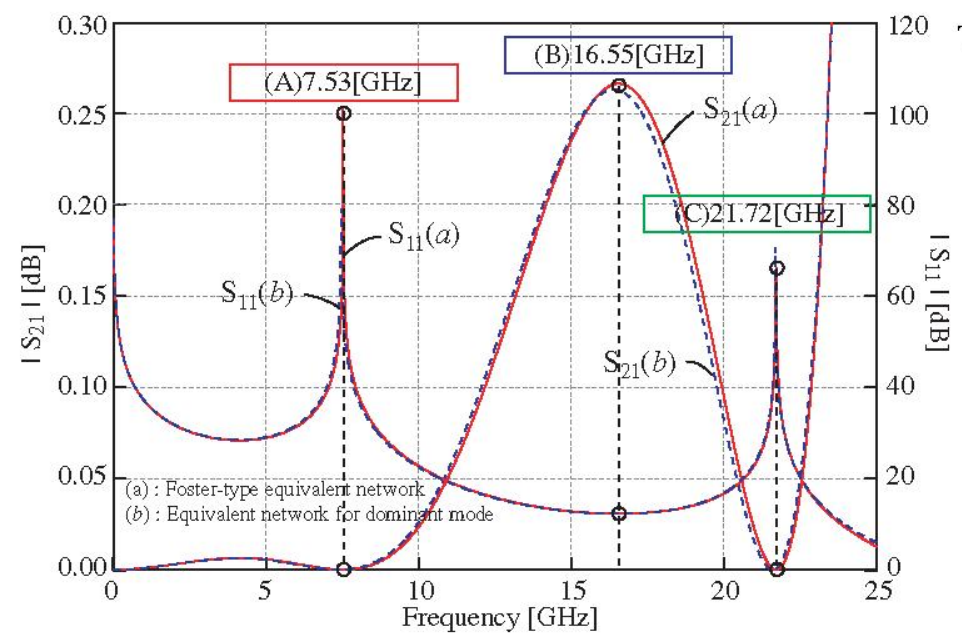

(a) Frequency characteristics for right-angle bend and the middle waveguide length $L=5.0[\mathrm{~mm}]$

Table 3 Transmission and reflection coefficient at frequency (A), (B) and (C)

\begin{tabular}{|c|c||c|c|}
\hline & $(A)$ & $(B)$ & $(C)$ \\
\hline$F[\mathrm{GHz}]$ & 7.53 & 16.55 & 21.72 \\
\hline$|\mathrm{S} 21|[\mathrm{dB}]$ & $3.50 \mathrm{E}-10$ & 0.267 & $1.02 \mathrm{E}-06$ \\
\hline$|\mathrm{S} 11|[\mathrm{dB}]$ & 100.930 & 12.254 & 66.290 \\
\hline
\end{tabular}

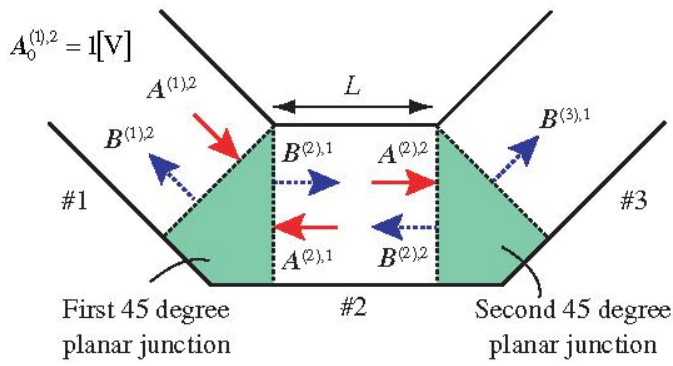

(b) Incident and reflected mode voltage
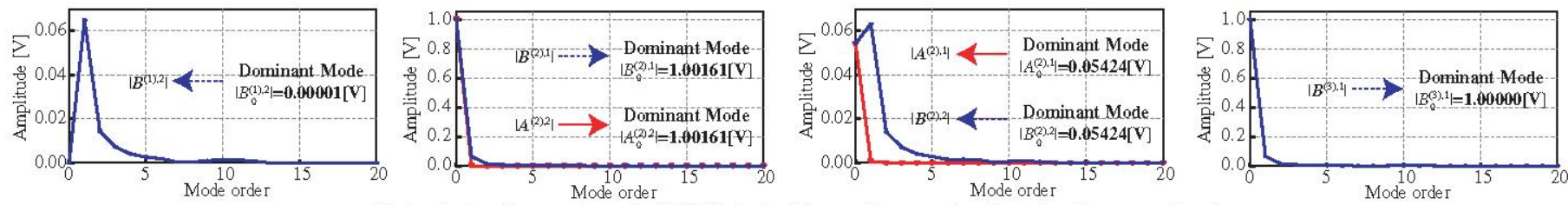

(c) Analysis of operation at $7.53[\mathrm{GHz}]$--incident voltage and reflected voltage amplitude

Fig.6 Characteristics of incident/reflected amplitude at each port vs mode order for unit incidence of dominant mode

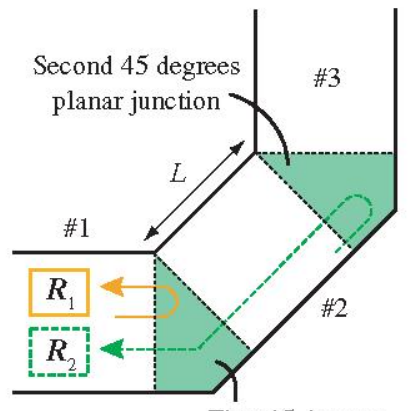

First 45 degrees planar junction

(a) Reflected component of $B^{(1), 2}$

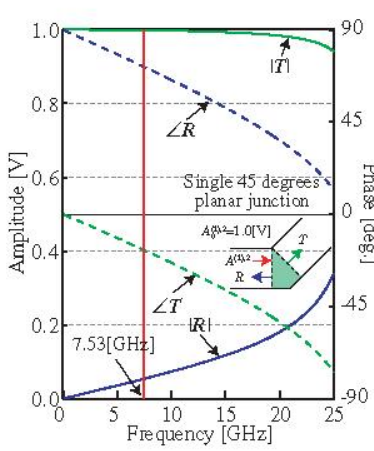

(b) Transmission and reflection of single 45 degrees planar junction

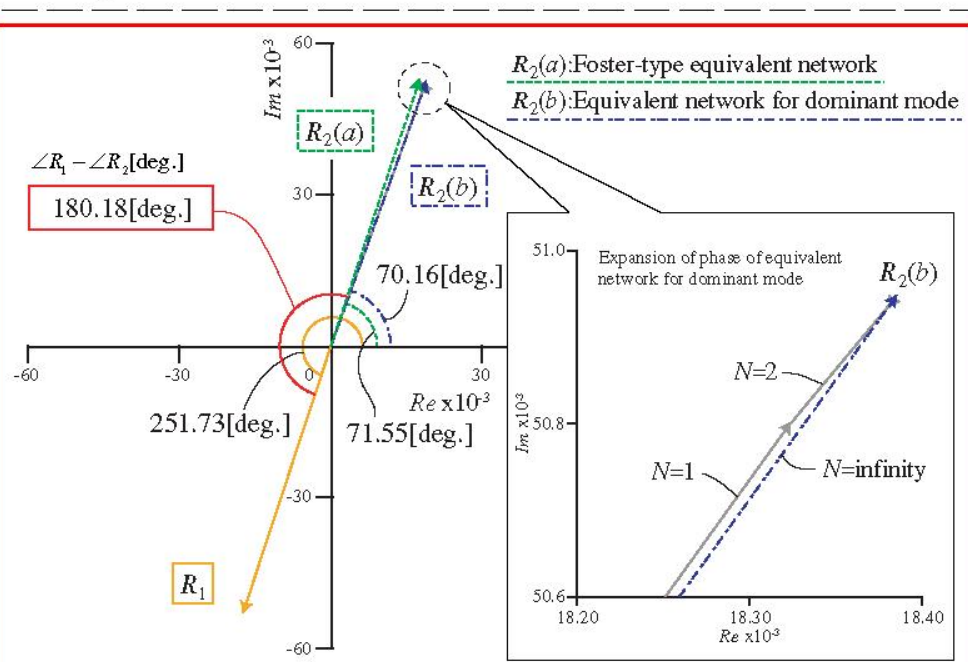

(A) $7.53[\mathrm{GHz}]$

Table 4 First reflection $R_{1}$, second reflection $R_{2}$ and total reflection(=vector sum of both) in amplitude and phase angle representaion at frequency (A), (B) and (C)

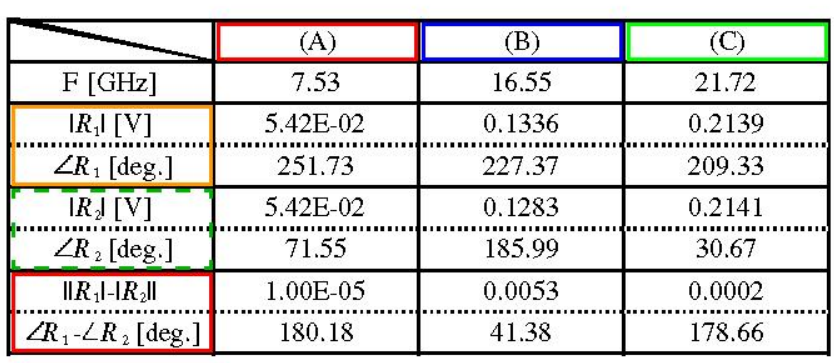

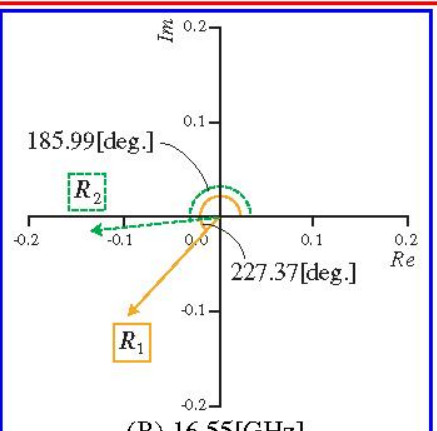

(B) $16.55[\mathrm{GHz}]$

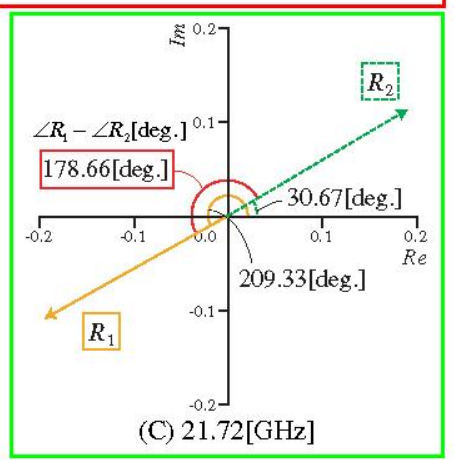

(c) Phase $R_{1}, R_{2}$ (Dominant Mode)

Fig.7 First reflection $R_{1}$ and second reflection $R_{2}$ at frequency (A),(B) and (C) for operational analysis of complete transmission at (A) and (C) 